\section{Towards Sustainable Urban Car- Parking Solutions: Exploring Effects of Parking Policies Using Spatial Regression Analysis}

Gl_Forum 2021, Issue 2 Page: 228 - 241 Full Paper

Corresponding Author: mengchin@g.nccu.edu.tw DOI: 10.1553/giscience2021_02_s228

\author{
Meng-Chin Tsai, Stephan van Gasselt and Tzu-Chin Lin \\ National Chengchi University, Taipei, Taiwan
}

\begin{abstract}
In urban policy development and transport planning, car parking has become a crucial topic, as an ill-designed system could lead to traffic congestion, safety problems, increased air pollution and other challenges, particularly in densely populated urban areas. Adjusting parking fees has been deemed the most effective tool to control parking behaviour for selected locations, in order to attract or repel drivers. However, in order to select target areas efficiently, and before any recommendation for fee adjustment is made, an analysis of the current parking situation and the identification of variables influencing parking behaviour are needed.
\end{abstract}

We present a spatial analysis targeted at parking demand and supply data gathered via road surveys by local authorities in Taipei City. The spatial analysis is complemented by a regression analysis to identify variables that influence parking behaviour.

Our approach examines the relationship between potential controlling and contributing factors, and we show the influence of variables in a specific example. Our aim is to provide a starting point for future policy development and pricing adjustment at a local level. This initial framework might provide a conceptual core for wider discussion and a tool for integrating other different scenarios in the future.

\title{
Keywords:
}

car parking policy, urban sustainability, spatial analysis, Geographically Weighted Regression (GWR)

\section{Introduction}

In urban transportation management, an effective parking policy is essential as increasing demand for mobility and denser traffic on the one hand need to be balanced with modern sustainable urban development concepts on the other (see e.g. Clements, 2019). The understanding is that parking behaviour can be controlled through effective price policies, which can control the overall traffic situation, and the attractiveness and safety of the neighbourhood or community in which the policy has been implemented. 
Other variables (in addition to parking fees) that describe and influence parking behaviour are factors such as cruising time spent for locating free parking spaces, travel time to final destination, and the purpose of the parking (Brooke et al., 2014; Brooke, 2016; Ma et al., 2013; Yun et al., 2009). Of these factors, parking fees are considered the most influential, as it has been shown that lower-priced parking generally results in higher occupancy levels of parking spaces. Free on-road parking, as one extreme, could lead to traffic congestion and thus longer journey times, and result in an overloaded parking system. Moreover, if all parking spaces are free of charge, society has to bear the cost, which might ultimately lead to a deterioration of the quality of the available parking and of the service provision (e.g., Shoup, 1997; 2020). While it may be straightforward to charge for car parking homogeneously across a city, a more detailed fee policy has the potential to help adjust local situations and to create a better balance across the city.

This very discussion took place in Taipei City (see Figures 1a and 1b), and in 2015 fees were introduced for on-street parking. Before the policy was implemented, people used to park on the streets, leaving their cars there for long periods (up to several months) without moving them at all). According to the Taipei City Parking Management and Development Office, the main goal of this policy is to increase the turnover of kerb-side parking spaces to improve parking efficiency. Pricing for car parking was developed according to the Taipei City Public Parking Lot Rate Autonomy Act, which stated that the management agency responsible for the parking spaces would set varying rates according to the region, traffic flow and time of day. In spite of the requirement to set parking fees, the city government's approach to investigating parking behaviour is based on traffic zones (see Figure 1b), which do not coincide with the pricing scheme of charged road segments. While the effects of car-parking policies have been studied in great detail over the years (e.g., Young, 1988; Feeney, 1989; Barter, 2012, 2015, 2018; Marsden, 2014; Biswas et al., 2017), the effect of this policy implementation has not so far been investigated in detail. It seems timely to carry out a preliminary analysis of the situation before and after the policy was implemented, using the available statistics and metrics.

a

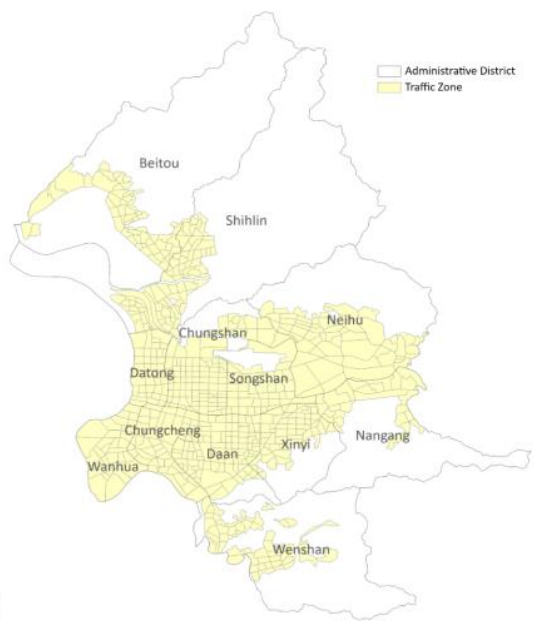

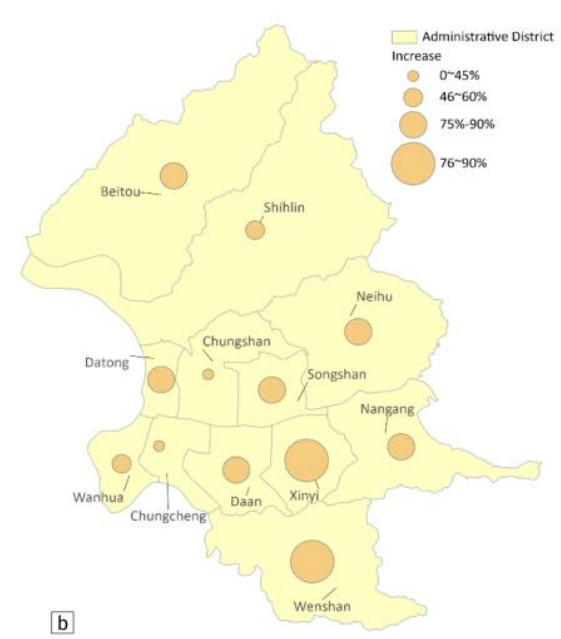

b

Figure 1: (a) Geographic distribution of urban districts and traffic zones in Taipei City; (b) districts with increased parking fees (city-wide car-parking policy introduced in 2015). 
The city of Taipei proper is home to approximately 2.65 million inhabitants, with over 7 million people in the Greater-Taipei metropolitan area, which comprises Taipei City, New Taipei and Keelung (MOI, 2021). Taipei City has an average population density of about 9,900 people per square kilometre. To put this into perspective, most capitals in Europe are home to about 3,000-5,000 inhabitants per square kilometre; Asian capitals vary considerably, with Seoul being relatively high, with 16,000 inhabitants per square kilometre.

As in many Asian capital cities, the car parking situation in Taipei is tense, with high demand and limited supply at all times (see Barter, 2012), and comparably high parking fees charged by private providers of car-parking spaces. According to a recent report, Taipei City (like Beijing) follows a 'moderate path' with 'modest parking standards' and the development of multiobjective parking management policies. These policies are aimed at 'serving wider urban and transport policy goals' rather than optimizing demand and supply, as in more conventional approaches (ADB, 2011).

Taipei City's government introduced fees for on-road parking in order to reduce lengthy stays in particular parking spaces, especially in residential areas. The aims were to improve the traffic situation and to contribute to a more sustainable living environment using local indicators and developments in the field of smart parking and neighbourhood traffic improvements (Chan, 2019; UN, 2015). A complementary aim of the city government has been to improve the demand to supply ratio of parking spaces. The new policy has addressed only part of Taipei's parking situation, and it is difficult to ascertain whether changes in parking behaviour are due simply to the introduction of parking fees.

Management decisions in relation to parking policy have often been criticized for not integrating various transportation variables (e.g., Young et al., 1991). There exists only limited research showing the picture before and after a parking policy has been introduced. As parking policies can have a number of effects also on local businesses, a broader understanding is needed before recommendations are made (Chaturvedi, 2012).

The driving question in this investigation is whether the change of policy has changed overall parking behaviour significantly, as parking behaviour is known to be influenced by a number of factors but predominantly by charge adjustments. Along with this question comes the challenge of identifying (1) factors or variables that influence the behaviour, and (2) metrics recorded before and after the policy implementation, which may provide clues. In summary, our aims were:

1. Identifying changes before and after policy implementation in 2015;

2. Identifying variables that influence parking behaviour;

3. Promoting discussions on metrics to be collected in the future, which might allow for a detailed assessment of the challenges faced.

In the following section, we discuss the methodological approach taken to address these aims and summarize the available statistical data that was used in this investigation (Section 2). In Section 3, we discuss results from an exploratory statistical analysis, and those of a spatial regression model that was used to identify contribution variables. Finally, we end with a summary, conclusion and a brief outlook. 


\section{Methods and Data}

In order to address the first aim, namely to identify changes before and after policy implementation in 2015, the first part of this work covers a spatial analysis that uses a set of conventional tools to identify local clustering, and the significance of any clusters and hot/ coldspots that might appear.

The second part of our research focuses on dependencies between a set of variables and a regression analysis to study the effects of these variables. This approach will provide insights into the second aim of the investigation, namely the identification of variables that influence parking behaviour; behavioural changes after the policy implementation might not be controlled by changes to parking charges alone. Both parts will highlight a number of challenges in response to the third aim, to identify open issues that need to be addressed further in subsequent work.

The basis of our investigation is data from the Parking Demand and Supply Surveys conducted between 2013 and 2017 for each traffic zone and road segment in Taipei City. The traffic zones in each administrative district were investigated for demand, supply, illegal parking, and both on- and off-road parking. There are 684 traffic zones in 12 administrative districts, supplying over 18,000 kerb-side parking places; half of these parking spaces require parking fees to be paid since the parking policy came into effect in 2015 (see Figures 1a and 1b).

The data from the Parking Demand and Supply Surveys include locational information and information about parking charges, in addition to the demand and supply data as represented by occupancy figures for both on- and off-road parking. All data were provided by the Taipei Parking Management and Development Office. In addition, demographic statistics were obtained from the Social and Economic Statistic Database of the Social and Economic Statistics Department, Ministry of the Interior, Taiwan. These data are complemented by data on urban zoning and land-use types, obtained from the Land Use Investigation for Taiwan, which was conducted by the National Survey Center in 2014. Additional data were derived during the spatial analysis using ESRI's commercial ArcGIS platform (Figure 2). 


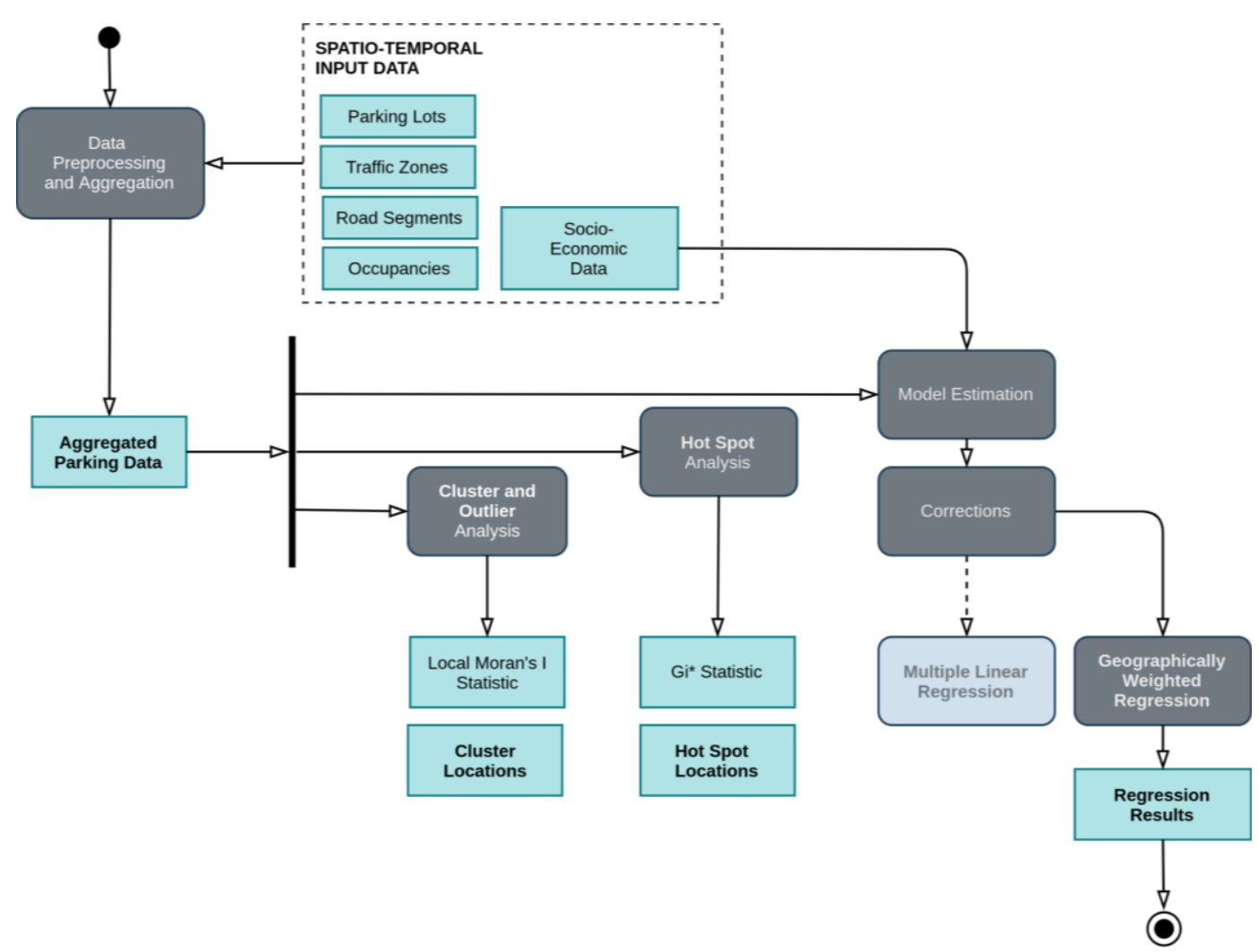

Figure 2: Data processing and analyses workflow as conducted in this investigation

Data pre-processing involves the aggregation of data from the street level to the level of the traffic zone, as street-level data are too granular to be merged with socio-economic variables. As most previous research has focused on data from a non-spatial perspective, we introduce a set of spatial analyses to obtain a better view of the data and the effects of policy on behaviour. Clustering is our first choice when it comes to identifying areas that could reveal significant effects after the policy implementation. Both Hot-Spot analysis (Getis \& Ord, 1992; Ord \& Getis, 1995) as well as Cluster and Outlier analyses (Anselin, 1995) were performed to identify correlations between pricing and location, both before and after policy implementation. More specifically, the Getis-Ord $G^{*}$ statistic was expected to reveal spatial clustering of higher and lower parking charges, while the Moran's I was calculated to reveal significant spatial autocorrelation (see Figure 2). We expected the combination of both sets of statistics to provide further quantifiable insights into the results of the policy implementation. This approach to better characterize the parking situation by identifying potential patterns with respect to charges and location is of an exploratory nature.

The main purpose of this study was to obtain insights into the relationships between variables that might influence parking behaviour, from external variables (such as socio-economic factors), to location-dependent variables (such as proximity of alternative parking spaces and amount of parking space in a neighborhood). These relationships are explored using (1) a 
Multiple Linear Regression (MLR) approach in the first step; they are further expanded upon using (2) a Geographically Weighted Regression (GWR) (Figure 2). Both MLR and GWR build on socio-economic variables that had been collected and integrated for each traffic zone area. They incorporated alternatives, such as the availability of off-road parking, which could potentially affect parking decisions.

In this study we collected, variables based on discussions in the research literature, and performed analyses to see whether parking behaviour is significantly affected by these variables. In order to obtain reliable results from the MLR, a number of tests and adjustments are required, including linearization of dependent and independent variables, and testing for multicollinearity and for homoscedasticity of error variances (Osborne and Waters, 2002; Uyanık and Güler, 2013) using the Breusch-Pagan test. For estimation of variables, the variables need to be tested for their relevance, using $\mathrm{R}$-square values for comparing predicted and actual values. Finally, the significance of regression results is tested using an F-test over the mean-square regressions and the mean-square error. From the first results of the spatial regression analysis, it could be observed that parking patterns were distributed heterogeneously, showing that error variance is not uniform. This heteroscedasticity causes problems in the regression. This led us to select a more appropriate regression model (see Figure 2). The Geographically Weighted Regression (GWR), as an extension to the MLR, renders the original model more sophisticated by allowing relationships between the independent and dependent variables to vary by locality (Brunsdon et al., 1996). The spatial weights of observations are collected based on the distances between each location. GWRestimated parameters are allowed to vary over space, which can be more suitable for analysis than arbitrarily-sized areas. It is known that GWR-estimated parameters have a better fit and offer better prediction in a scenario showing significant spatial heterogeneity (Brunsdon, et al., 1996; Fotheringham et al, 1999; Fotheringham et al, 2002).

The ratio of parking demand to supply is used as a descriptive index in this work. This ratio is the number of spaces occupied by cars (the demand) to the total number of off- and on-road parking spaces available in each traffic zone (supply). A small ratio indicates that the parking spaces are not being used efficiently, which means that the parking situation is relaxed but that there is an oversupply of parking space. Ratios in 2014 and 2015 were classified into five different categories to describe in detail the availability of parking spaces in each traffic zone. Ratios close to 1 reflect balanced demand and supply. Ratios between 1 and 1.25 indicate saturated supplies, and drivers may spend time cruising in search of a parking space or have to endure long waiting times. If the ratios are over 1.25, the parking system should be adjusted to avoid problems for the transport system as a whole.

\section{Results and Discussion}

In order to address our first aim, we mapped the classified demand-to-supply ratio (see Figure 3 ) and the distribution of charges (Figure 4) before and after policy implementation. In addition, we performed cluster and hotspot analyses to identify distribution characteristics that required further investigation. 
In Figure 3, it can be seen that the overall situation after the introduction of the policy shifted towards a higher demand in a number of traffic zones, which puts more strain on available parking, but also helps optimize land use. Overall, the distribution shows subtle changes, with a number of potential clusters of higher ratios of demand to supply.
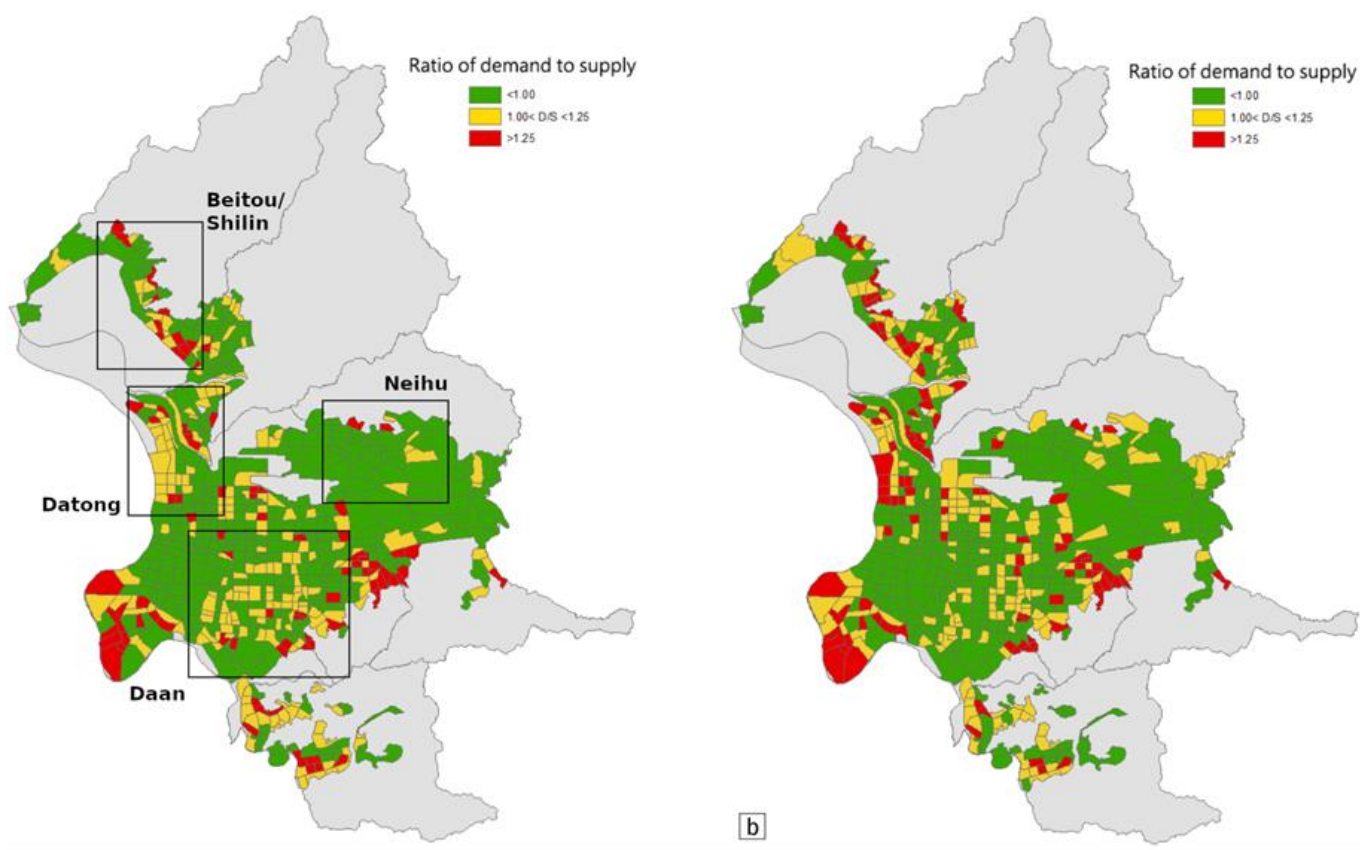

Figure 3: Demand to Supply Ratio for traffic zones in Taipei City (a) before parking-policy implementation in 2015, and (b) after implementation. Black boxes indicate (named) areas where there was significant change between the two periods (see also Figure 1 for reference)

The spatial distribution of fees (Figure 3) shows a significant change towards charging for parking throughout the city area, and towards lowering the parking charges. High charges remain within the city centre, while the second-highest charges are seen mainly in the northern and southern fringes, which are increasingly popular residential areas. 
Tsai et al
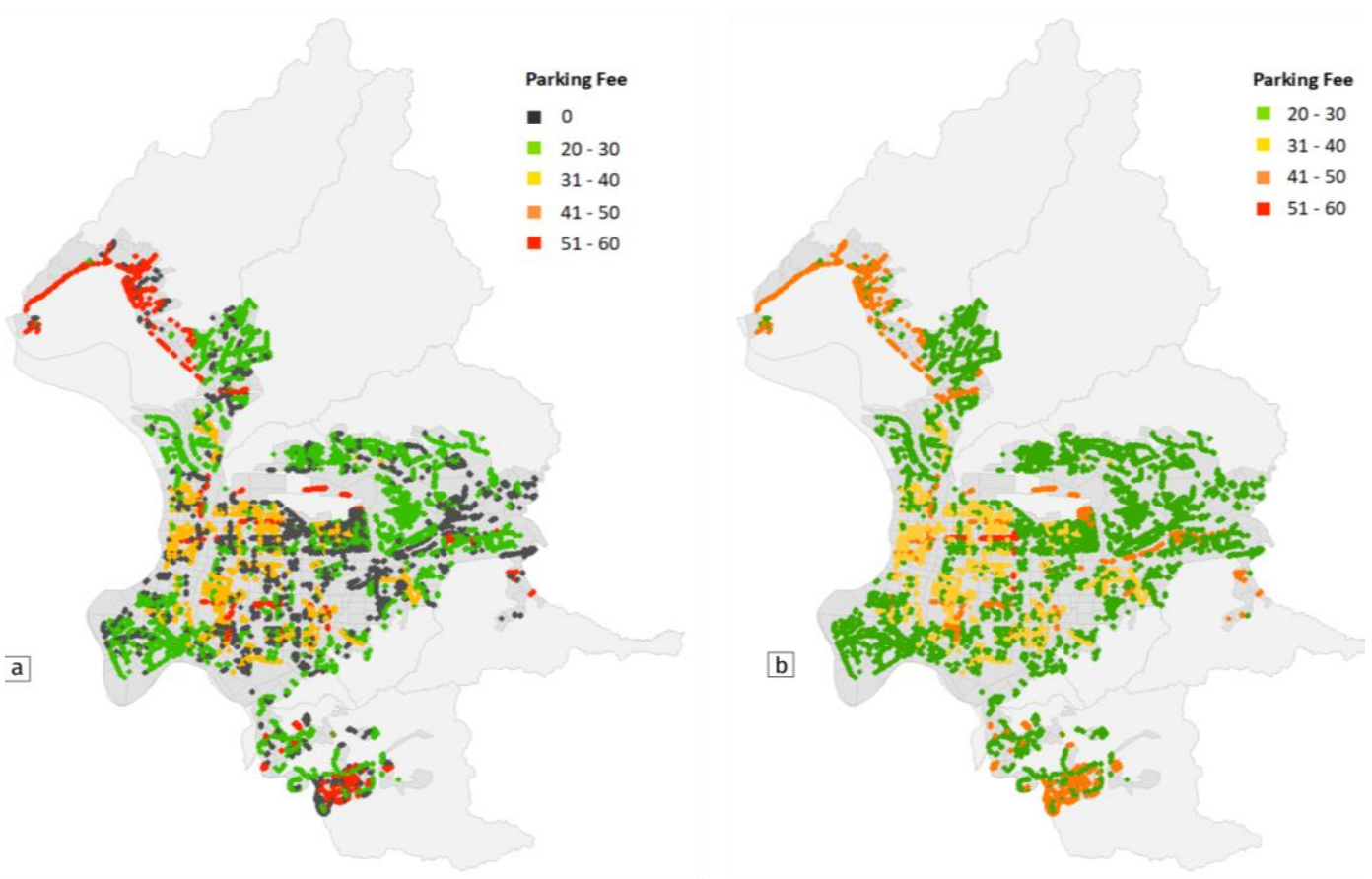

Figure 4: Distribution of kerb-side parking fees across districts and traffic zones in Taipei City, (a) before and (b) after policy implementation. (Fees in New Taiwan Dollars; 1 NTD $=0.03$ EUR at the time of writing.)

The Cluster and Outlier analysis (Figure 5a) shows clear outliers in four districts where parking fees are higher. A small number of parking spaces are charged at a higher rate compared to surrounding ones (red areas in Figure 5a); in other traffic zones, some kerb-side parking spaces are charged less than in the surroundings (blue areas in Figure 5a). Both groups represent outliers. The effects of policy adjustments are well represented in these heterogeneous patterns indicating outliers. The distribution of outliers provides valuable information about location and direction of price adjustment. 

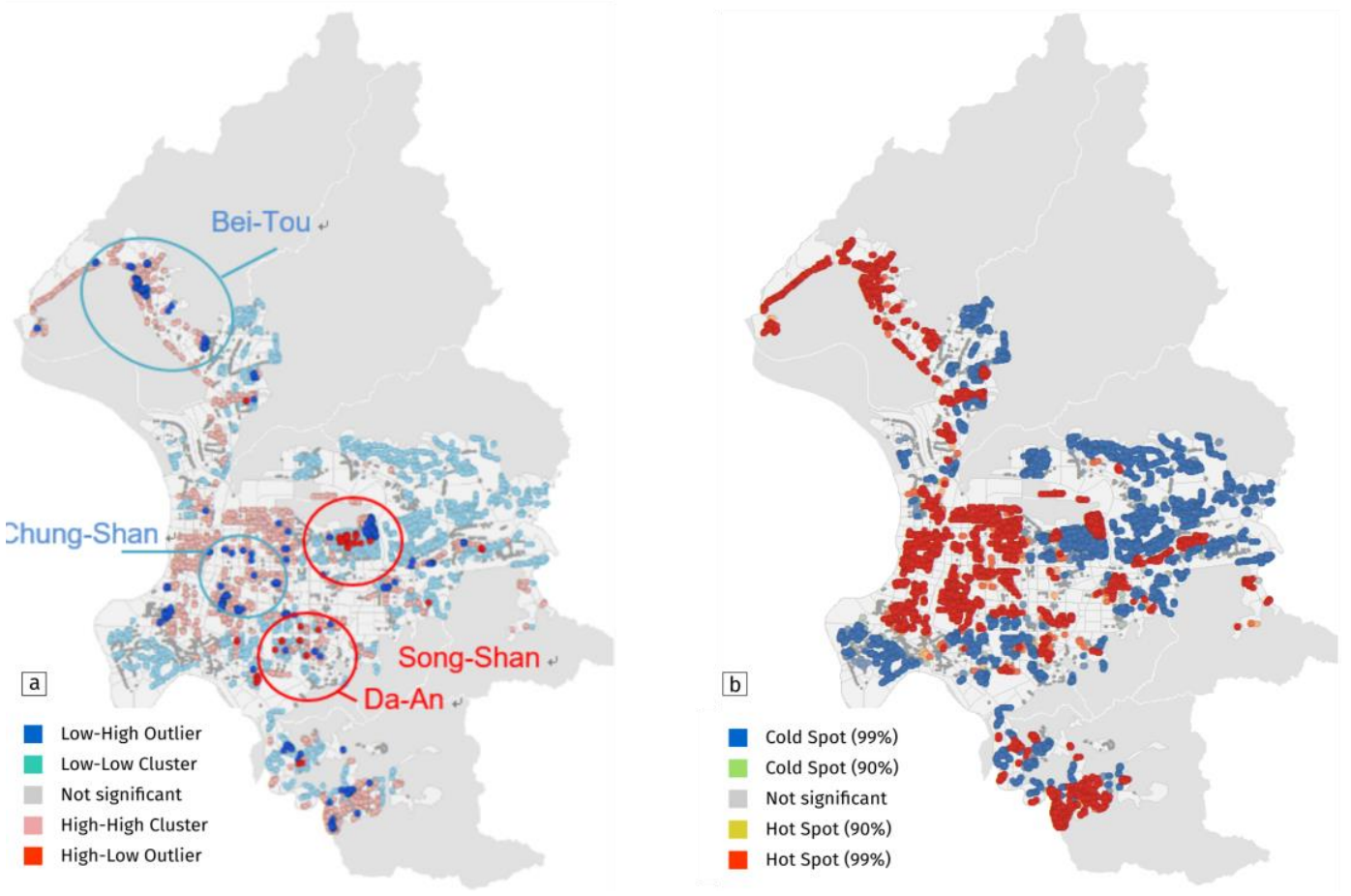

Figure 5: Distribution of (a) parking fee clusters and outliers, and (b) cold- and hotspots at traffic-zone level in Taipei City

In Figure 5b, kerb-side spaces charging higher fees appear as hotspots (in red) within areas of generally lower fees. The hotspots are predominantly in the western and central areas of the city. In contrast, outliers marked in Figure $5 \mathrm{a}$ are coldspots within their higher-charge environments. We can only speculate as to the reasons for these changes. While the local effect is likely to be to draw parking behaviour towards coldspots, the change might also increase pressure in the already tense inner-city traffic situation.

A secondary aim of our research was to assess the external (i.e. non-policy related) variables that were recorded along with on-street assessment of parking behaviour, in order to identify (a) the impact of these external variables on behaviour, and (b) their suitability as potential indicators in future assessments. In order to better understand dependencies, an initial MultiLinear Regression analysis was performed using spatial and non-spatial socio-economic variables (e.g., household statistics). 

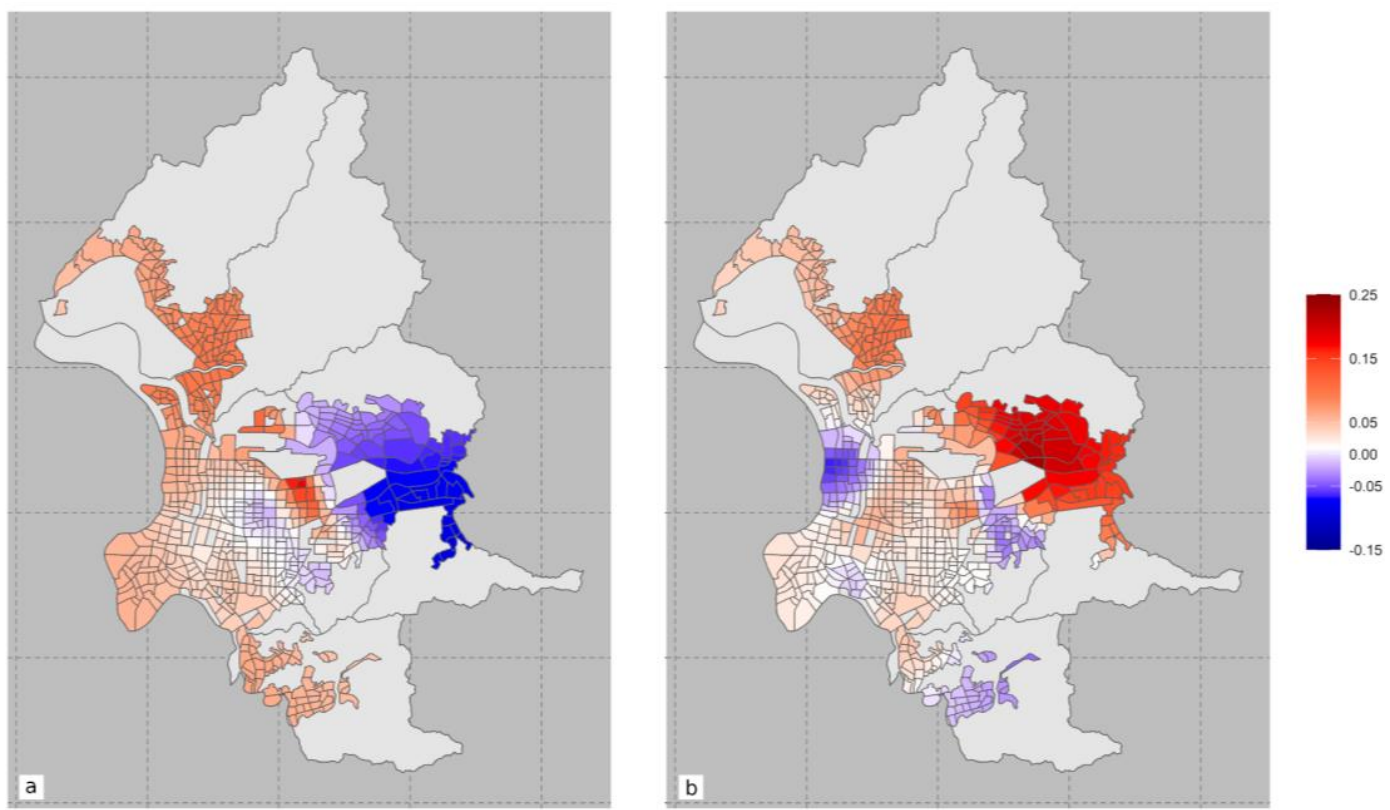

Figure 6: Coefficient results for the Geographically-Weighted Regression for the variable 'illegal parking (of cars)', (a) before and (b) after implementation of the new policy

Two approximate models were compared to determine which of them shows a larger effect with respect to parking-space occupancy. In the first model, numbers of on-road and off-road parking spaces were considered, accompanied by factors including land-use characteristics, and social properties such as number of households. The choice of variables was based on published studies - i.e., we selected the same variables as those found in other studies to be responsible, in the main, for drivers' parking choices. Before the policy was introduced, drivers' choice of parking spaces was affected by the total supply of on-road parking spaces - i.e. they could choose parking spaces anywhere along the road. After the introduction of the policy, residents in a traffic zone could be negatively affected by increased parking fees as their choice was now limited.

Population, number of households, and the proportion of residential land-use area were removed from the second model, due to multicollinearity effects. This new regression model has a higher explanatory power. In addition, the homoscedasticity problem that was observed with the first model was improved. Carrying out the same analysis after policy implementation revealed that drivers still preferred to occupy on-road parking spaces. Parking behaviour changed only slightly after the introduction of the new policy (away from on-street parking), indicating that drivers' preferences will stay much the same, despite a rise in the average parking fee in a traffic zone.

The traditional MLR model shows a significant heteroscedasticity problem, which could be overcome by a regionally weighted approach, such as a geographically weighted regression (GWR). The GWR makes use of the same variables as MLR model 2, analysing spatial patterns and impacts at local levels. As one example of the GWR analysis, correlation coefficients for 
illegal parking are shown in Figure 6, for the periods before and after the policy implementation. Since on-road parking spaces are generally charged, some drivers will look for cheaper alternatives, and some will even park illegally along roads where parking is not allowed. Using the traditional regression model, it is not possible to identify the areas where this situation affects occupancy levels. Model 2 shows another effect, in which a decrease of illegal parking by 0.028 correlates with freeing one more kerb-side space for occupation (Figure 6). If we compare the situation before and after the introduction of the policy for each traffic zone, we see that drivers in each traffic zone show different preferences with regard to occupying the kerb-side spaces. Before the policy was implemented, they showed less preference for off-road supplies; after the average parking charge in the traffic zone was raised, they tended to park in kerb-side spaces. Also, illegal parking increased after the introduction of the policy.

Table 1: Comparison of Multiple Linear Regression (MLR) and Basic Geographically Weighted Regression (GWR) results before and after policy implementation. (RSS: residual sum of squares.)

$\begin{array}{lllll} & \text { Before Policy } & & \text { After Policy } & \\ & \text { MLR } & \text { Basic GWR } & \text { MLR } & \text { Basic GWR } \\ \text { R2 } & 0.9362 & 0.9558 & 0.9354 & 0.9573 \\ \text { RSS } & 142,091.4 & 98,514.29 & 167,244.5 & 110,472\end{array}$

\section{Summary and Conclusions}

Considering today's dynamic urban environment and increasing sensitivity towards urban sustainability and mobility (Stubbs, 2002; Banister, 1998), parking policies need to be adaptive and reviewed on a regular basis to respond to the changing demands. The assessment of parking policies must be conducted using tools that can be readily applied, with indicators and metrics that can describe the actual situation (e.g., Barter, 2011). However, assessing parking situations in a uniform way across different traffic zones (or analogues thereof) poses a number of challenges, with respect to addressing not only the temporal dynamics, but also the influence of variables that are spatially as well as temporally dynamic.

The impact of the Taipei City government's new parking policy was assessed through a series of spatial analyses which aimed to gain a better understanding of the situation before and after the policy's introduction in 2015. A further aim was to (potentially) identify variables within their spatial contexts that might prove useful for future policy reviews and developments in response to changing traffic demands.

Despite many strategic improvements and a wider availability of tools to manage parking and develop car-parking policies, management decisions do not always address the actual dynamics of parking behaviour, due to the complexity of interdependencies, the lack of representative and meaningful (or even consistent) data, and new objectives that have to be integrated for a more sustainable urban environment. An analysis of the parking data that is currently available 
for investigating parking in Taipei City showed pronounced clusters of parking places with high parking charges but limited demand as derived from hotspot, cluster and outlier analyses.

In this study, spatial analyses were complemented by a Multiple Linear Regression (MLR) in order to identify variables that influence parking behaviour within a traffic zone, and to gain a better understanding of the effectiveness of the policy employed. It turned out that the choice of specific variables (which was based on a literature review) and the application of an MLR led to the appearance of pronounced multicollinearity effects. These effects had to be addressed by adapting the regression model, removing variables originally deemed relevant. A Geographically Weighted Regression (GWR) emerged as a more accurate approach to capture drivers' responses to the policy adjustment.

Before the policy was implemented, drivers reacted less to off-road availability of spaces; following a rise in the average parking charge in a traffic zone, they tended to use the kerbside parking spaces. In addition, the use of illegal parking spaces increased after the introduction of the policy.

For all traffic zones across the city, drivers show a preference for on-road parking, which might be related to their daily habits. For every 7 to 10 on-road parking spaces, there are about 10 drivers who would prefer to park at an indoor location.

According to the empirical results, parking behaviour has changed since 2015. However, in most districts, drivers are still willing to occupy kerb-side spaces even though the parking fees have risen. Hence, it seems safe to state that targeting charges in an attempt to adjust parking behaviour might have limited success in improving a parking situation or regulating traffic. It could also be seen in a few districts that further parking choices (e.g. off-road parking, or carparks attached to specific buildings or workplaces) can improve the problem of the high demand for on-road parking. Introducing more restrictions and customizing the parking policy for individual administrative districts may be other feasible options. Since Taipei has introduced experimental smart parking in several districts, new real-time data could soon be available that would provide higher temporal resolution of drivers' responses to parking adjustments.

The initial analytical procedure presented here could support discussions on policy impact, and enhance understanding of the relationships between parking behaviour and the attractiveness of locations. Ultimately, the approach could influence the design of parking infrastructure in order to address new needs in the context of sustainable city development.

\section{Acknowledgements}

The authors wish to thank the organizers and three anonymous reviewers for their comments and critical questions that have helped to improve the manuscript. We also wish to thank Mary Rigby for her extraordinarily detailed and helpful copyediting. 


\section{Reference}

ADB (2011). Parking policy in Asian cities. Asian Development Bank, Mandaluyong City, Philippines; $112 \mathrm{pp}$.

Anselin, L. (1995). Local Indicators of Spatial Association - LISA. Geographical Analysis 27(2): 93115.

Banister, D. (1998). Barriers to the implementation of urban sustainability. International Journal of Environment and Pollution, 10(1): 65-83, doi:10.1504/IJEP.1998.002231.

Barter, P.A. (2011). On-Street Parking Management - An International Toolkit. Sustainable Urban Transport Technical Document \#14; Deutsche Gesellschaft fuer Internationale Zusammenarbeit, Federal Ministry for Economic Cooperation and Development; 120 pp.

Barter, P.A. (2012). Off-street parking policy surprises in Asian cities, Cities, 29(1): 23-31, doi: 10.1016/j.cities.2011.06.007.T

Barter, P.A. (2018). Parking Policies in Asian cities: Conventional but instructive. In D. Shoup (Ed.), Parking and the City (pp. 161-170). New York, NY: Routledge.

Barter, P.A. (2015). A parking policy typology for clearer thinking on parking reform, International Journal of Urban Sciences, 19:2, 136-156, doi: 10.1080/12265934.2014.927740.

Biswas, S., Chandra, S. and Ghosh, I. (2017). Effects of On-Street Parking In Urban Context: A Critical Review, Transportation in Developing Economies, 3:1-14.

Brooke, S. (2016). Factors influencing urban on-street parking search time using a multilevel modelling approach, $\mathrm{PhD}$ thesis, Architecture, Building and Civil Engineering, Loughborough University, UK.

Brooke, S., Ison, S. \& Quddus, M. (2014). On-Street Parking Search, Journal of the Transportation Research Board, 2469:65-75.

Brunsdon C., Fotheringham A. S. \& Charlton M. E. (1996). Geographically weighted regression: a method for exploring spatial nonstationarity, Geographical Analysis 28:281-298; doi:10.1111/j.1538-4632.1996.tb00936.x

Chan, C. C. (2019). Global Perspective: Taipei in Motion - A Proposal of Voluntary Local Review for SDG Progress; presentation on 18 March 2019, New York.

Chaturvedi M., 2012, Parking in Balance: A Geospatial Analysis of Efficiency of the Parking System of Enschede, The Netherlands, University of Twente Faculty of Geo-Information and Earth Observation (ITC).

Clements, R. (2019). Parking and the City, Planning Theory \& Practice, 20:3, 456-465, DOI: 10.1080/14649357.2019.1627120

Feeney, B. P. (1989). A review of the impact of parking policy measures on travel demand; Transportation Planning and Technology, 13:4, 229-244, doi: 10.1080/03081068908717403.

Fotheringham A. S. \& Brunsdon C. (1999). Local forms of spatial analysis; Geographical Analysis, 31(4):340-358.

Fotheringham A. S., Brunsdon C. \& Charlton M. (2002). Geographically Weighted Regression: The Analysis of Spatially Varying Relationships, 284 pp., Wiley.

Getis, A. and J.K. Ord (1992). The Analysis of Spatial Association by Use of Distance Statistics. Geographical Analysis 24(3).

Ma, X., Sun, X., He, Y. \& Chen, Y. (2013). Parking Choice Behavior Investigation: A Case Study at Beijing Lama Temple. Procedia - Social and Behavioral Sciences, 96:2635-2642.

Marsden, G. (2014). Parking Policy, Parking Issues and Policies. Transport and Sustainability, 5: 11-32. doi:10.1108/S2044-994120140000005016.

MOI (2021). Taiwan Ministry of the Interior. Statistical Yearbook of Interior. Internet: https://www.moi.gov.tw/english (accessed 2021-01-25).

Ord, J.K. and A. Getis (1995). Local Spatial Autocorrelation Statistics: Distributional Issues and an Application. Geographical Analysis 27(4). 
Osborne J. \& Waters E. (2002). Four Assumptions of Multiple Regression that Researchers Should Always Test. Practical Assessment, Research \& Evaluation, 8(2); doi: 10.7275/r222-hv23.

Shoup D. (1997). The High Cost of Free Parking, Journal of Planning Education and Research 17:320.

Shoup, D. (2020). The Pseudoscience of Parking Requirements. 8 pp., Zoning Practice, American Planning Association, 2, Practice Parking Reform.

Stubbs, M. (2002) Car Parking and Residential Development: Sustainability, Design and Planning Policy, and Public Perceptions of Parking Provision. Journal of Urban Design, 7(2): 213-237. doi: 10.1080/1357480022000012249.

UN (2015). Transforming our World: The 2030 Agenda for Sustainable Development. A/RES/70/1, 41 pp., United Nations, Geneva.

Uyanık G. K. \& Güler N., (2013). A Study on Multiple Linear Regression Analysis, Procedia - Social and Behavioral Sciences, 106:234-240.

Young, W. (1988). A review of parking lot design models - Foreign summaries; Transport Reviews, 8(2): 161-181. doi: 10.1080/01441648808716682.

Young, W., R. G. Thompson \& M. A. P. Taylor (1991). A review of urban car parking models. Transport Reviews, 11(1), 63-84. doi:10.1080/01441649108716773.

Yun, M., Lao, Y., Ma, Y. \& Yang, X. (2009). Optimization Model on Scale of Public Parking Lot Considering Parking Behavior; Eighth International Conference of Chinese Logistics and Transportation Professionals (ICCLTP), 2692-2699. doi:10.1061/40996(330)398. 